\title{
Echtzeitholografie mit BSO-Kristall zum Messen der Schichtdickenänderung beim Aushärten von Zwei-Komponenten-Klebstoffen
}

\author{
B.Pfister, H.J.Tiziani \\ Institut für Technische Optik, Universität Stuttgart \\ Pfaffenwaldring 9, D-7000 stuttgart 80
}

\section{Einleitung}

Bei Präzisions-Klebeverbindungen empfindlicher Bauteile können infolge der Schichtdickenänderung des Klebstoffs beim Aushärten (Schwinden) neben maBlichen Veränderungen auch erhebliche Spannungen und daraus resultierende unzulässige Bauteildeformationen auftreten.

Um die Schichtdickenänderung quantitativ erfassen zu können, sind insbesondere beruhrungslos messende Verfahren geeignet, da die Messungen wăhrend des Aushärtens des Klebstoffes, d.h. in seiner "flüssigen Phase erfolgen müssen.

Unter bestimmten noch zu erörternden Voraussetzungen bietet sich die Echtzeitholografie an, diese MeBaufgabe zu lösen.

\section{MeBverfahren}

Es hat sich gezeigt, daB die Gesamtverformung der Klebstoffschicht während des Aushärtens den Meßbereich der Holografie um ein Mehrfaches übersteigt. Der gesamte Aushärtevorgang kann somit nicht mit dem ersten Hologramm (Nullhologramm) verglichen werden. Aus diesem Grunde muß eine Vielzahl von Einzelhologrammen möglichst lückenlos (ohne Informationsverlust) aneinandergereiht werden.

Die aus den Einzelhologrammen errechneten Relativverformungen können dann zur Gesamtverformung aufsumiert werden.

Da die Schichtdickenänderung $\Delta s(t)$ nach dem Mischen der beiden Komponenten Binder und Härter ein stetiger, nach einer Exponentialfunktion verlaufender Vorgang ist, resultiert vor allem für die Anfangsphase der Aushärtung die Forderung nach sehr kurzen Taktzeiten ( $\Delta \mathrm{T}<1 \mathrm{~min}$ ) fur das Echtzeit-Holografieverfahren. Wobei wăhrend $\Delta T$ das Hologramm belichtet und ausgewertet werden muß.

Die erforderlichen kurzen Taktzeiten können dann erreicht werden, wenn die Echtzeitholografie kombiniert wird mit einem Wismut-Siliziumoxid (BSO)-Kristall als Hologrammspeicher sowie einer automatischen Hologrammauswertung nach der Phasenshiftmethode. 
BSO-Kristalle als Hologrammspeicher

Bei der Hologrammspeicherung im BSO-Kristall wird der lineare elektrooptische (Pockels-) Effekt ausgenutzt.

Infolge der Fotoleitfähigkeit des BSO-Kristalls entsteht bei der Belichtung ein Raumladungsfeld entsprechend der Intensitatsmodulation des aus der Interferenz von objekt- und Referenzwelle resultierenden Mikrointerferenzstreifenmusters. Der elektrooptische Effekt bewirkt dann eine dem Raumladungsfeld entsprechende räumliche Modulation der Brechzahl (Phasenvolumenhologramm) $/ 1 /, / 2 /$. Dazu werden freie Ladungsträger an Orten konstruktiver Interferenz in das Leitungsband gehoben, die dann bevorzugt an orten destruktiver Interferenz getrappt werden.

Die Elektronendrift im BSO-Kristall und damit seine Empfindlichkeit labt sich durch Anlegen eines konstanten transversalen elektrischen Feldes (ca. $6 \mathrm{kV} / \mathrm{cm}$ ) erheblich erhohen. Dann beträgt die Empfindichkeit im blau-grünen Spektralbereich etwa $0,3 \mathrm{~mJ} / \mathrm{cm}$, für einen Beugungswirkungsgrad von $18 / 1 /$.

Da sich das Brechzahlgitter bereits wathrend der Hologrammbelichtung aufbaut, ist der BSO-Kristall besonders geeignet für die Echtzeitholografie. Er ist, zum großen Vorteil für die Praxis, beliebig oft löschbar, also wiederverwendbar; bei langzeitigem praktischem Einsatz sind keine Ermidungserscheinungen aufgetreten.

\section{Automatische Hologrammauswertung}

Das oben beschriebene holografische RelativmeBverfahren ist mit einem BSO-Kristall nur dann realisierbar, wenn die Einzelhologramme wahrend der minimalen Taktzeit von $\Delta T<1$ min ausgewertet werden können.

Dies 1st möglich mit einer rechnergestüzten Hologrammauswertung nach der Phasenshiftmethode /3/. Damit konnen Verformungen vorzeichenrichtig aus drei Hologrammen verschiedener aber bekannter Phasenbeziehung berechnet werden.

Die Phasenverschiebung wird hier mit einem Piezospiegel im Referenzstrahl vorgenommen, sie betrăgt jeweils $90^{\circ}$.

Bild 1 zeigt schematisch die verwendete Holografieanordnung mit BSOKristall (8) und rechnergestlitzter Hologrammauswertung.

Die für die Berechnung der Verformung erforderlichen drei phasenverschobenen Hologramme werden nacheinander uber eine TV-Kamera im Bild- 
speicher des Rechners abgespeichert. Aus den Intensitäten $I_{1}(x, y)$, $I_{2}(x, y), I_{3}(x, y)$ der drei Hologramme kann die Interferenzphase $\phi(x, y)$ berechnet werden und daraus die Verformung für jeden objektpunkt.

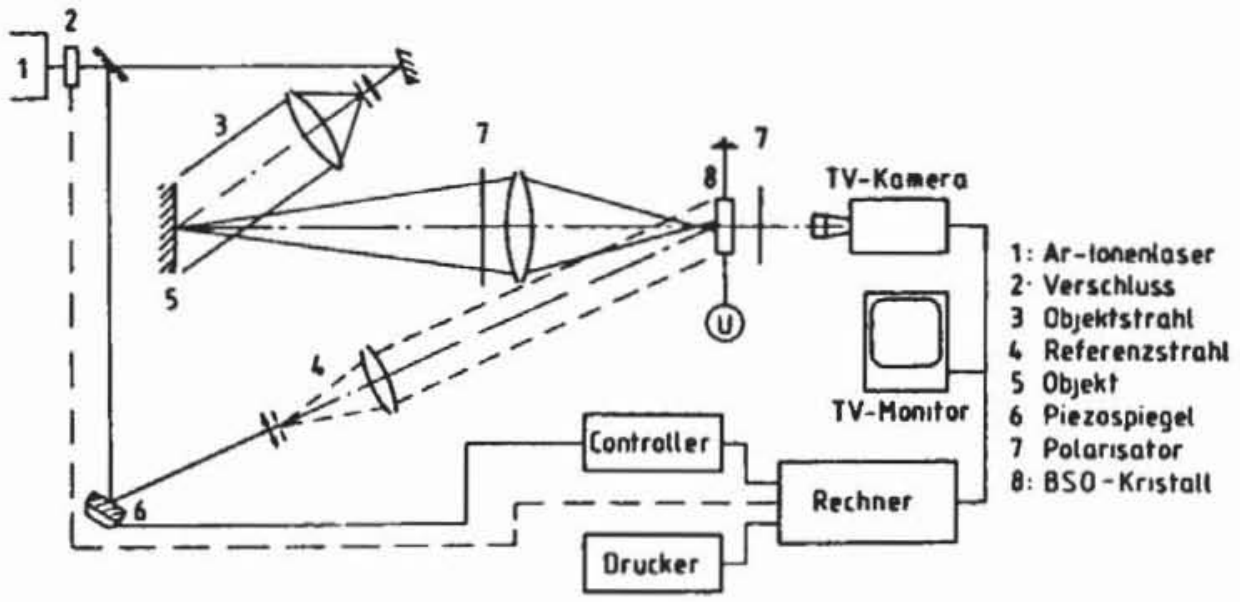

Bild 1. Versuchsanordnung Echtzeitholografie mit BSO-Kristall und automatischer Hologrammauswertung

\section{Ergebnisse}

Die Messungen wurden an einer keilförmigen Klebstoffschicht vorgenommen, bei einer Anfangsschichtdicke von $0,15 \mathrm{~mm}$ ( $\max$. Keildicke). Um Adhäsionskräfte und daraus resultierende Verformungen an der kreisförmigen Berandung $(25 \mathrm{~mm}) \mathrm{zu}$ minimieren, war die Berandung aus Teflon angefertigt. Da die Holografie diffus reflektierende Oberflächen verlangt, mu $B$ der relativ transparente Klebstoff mit einer dünnen mattweiBen Lackschicht liberzogen werden.
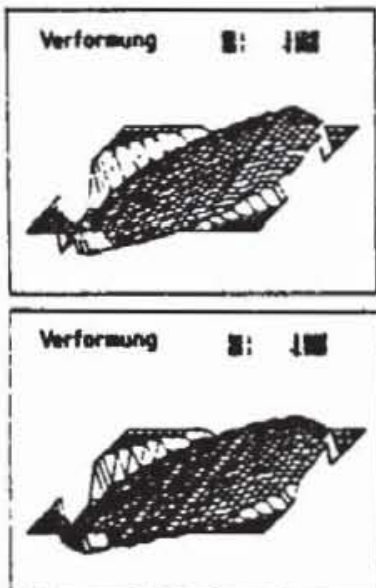
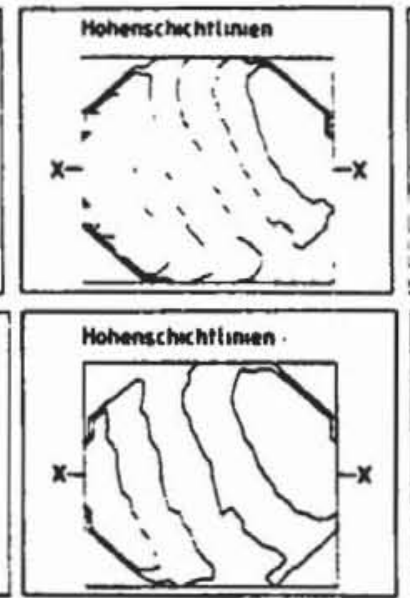
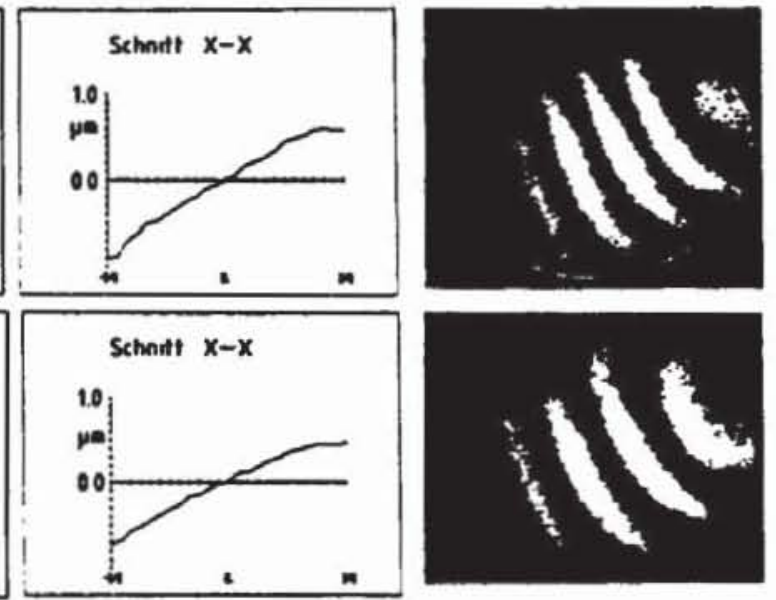

Bild 2. Automatisch ausgewertete Echtzeithologramme "Aushärten eines Zwei-Komponenten-Klebstoffs", a) berechnete Verformung, b) aus der Verformung berechnete Höhenschichtlinien, c) Schnitt durch die Deformation, d) rekonstruierte Hologramme 
Bild 2 zeigt beispielhaft zwei zeitlich aufeinanderfolgende, automatisch ausgewertete Hologramme im Verlauf eines Aushärtevorganges.

Aufgrund der keilformigen klebstoffechicht ergeben sich nahezu parallel verlaufende Interferenzstreifen. Es handelt sich somit um eine Neigung der Oberfläche um die Stelle des Keils mit der Dicke Null, uberlagert von einer schwachen Durchbiegung der Fläche.

In Bild 3 sind für einen Aushärtevorgang die relativen Verformungen von 35 Einzelhologrammen zur Schichtdickenänderung $\Delta 8$ aufsummiert, für einen Zeitraum von $t=39 \mathrm{~min}$ bis $t=250 \mathrm{~min}$ (nach dem Mischen von Binder und Härter) und einer Anfangsschichtdicke von $0,15 \mathrm{~mm}$.

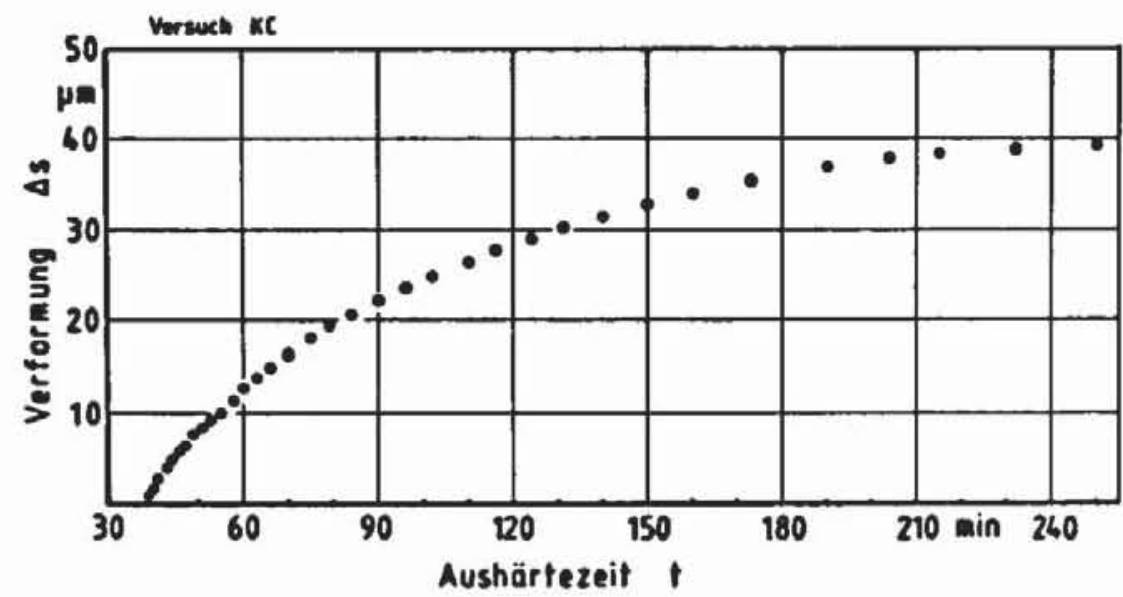

Bild 3. Schichtdickenänderung $\Delta_{s}(t)$ eines zwei-Komponenten-Klebstoffs, holografisch gemessen in Echtzeit, ausgewertet mit einer automatischen Hologrammauswertung

Nach 250 min entstehen im rekonstruierten Hologramm auch nach langerer Wartezeit (ca. $20 \mathrm{~min}$ ) keine Interferenzstreifen mehr, also auch keine Verformung und somit kein weiteres Schwinden der Klebstoffschicht. Die maximale Schichtdickenänderung beträgt nahezu $40 \mu \mathrm{m}$ nach $t=250 \mathrm{~min}$, die klebstoffschicht schwindet um ca. 26 \&, eine GröBenordnung, die bei präzisen Klebeverbindungen nicht vernachlässigbar ist.

\section{Literatur}

/1/ H.J.Tiziani

Real-time metrology with BSO crystals, Optica Acta 29(1982), 469

/2/ F.Holler, H.J.Tiziani

A spatial light modulator using BSo crystals, Opt.Commun.58(1986)

/3/ B.Dörband

Die 3-Interferogramm-Methode zur automatischen Streifenauswertung in rechnergesteuerten digitalen $\mathrm{zweistrahlinterferometern}$

Optik No.2 (1982), 161 